\title{
Abordagem das capacitações: uma discussão sobre desenvolvimento e estado
}

Capabilities approach: a discussion on development and state

\author{
Giana Silva Giacomelli \\ gianagiacomelli@gmail.com \\ Universidade Federal de Santa Maria (UFSM) \\ Aline Zulian \\ alinezulian@hotmail.com \\ Universidade Federal de Santa Maria (UFSM) \\ Paulo Ricardo Feistel \\ prfeistel@yahoo.com.br \\ Universidade Federal de Santa Maria (UFSM)
}

\begin{abstract}
Resumo: As temáticas que envolvem o desenvolvimento econômico e social têm recebido grande atenção na atualidade. No decorrer da história, são diferentes abordagens, conceitos e medidas adotadas para analisar o desenvolvimento das nações. Dentre as visões mais abrangentes sobre o assunto, está a abordagem de Amartya Sen, que adota uma visão multidimensional do tema, defende que o desenvolvimento abrange muito mais do que o crescimento econômico, incluindo as liberdades e a expansão das capacitações individuais. Por meio da utilização conceitual proposta por Sen, que considera o desenvolvimento como um processo de expansão das capacitações dos indivíduos, este artigo tem como objetivo promover a discussão sobre o papel do Estado no processo de desenvolvimento.
\end{abstract}

Palavras-chave: Estado e Desenvolvimento; Abordagem das capacitações; Amartya Sen

\begin{abstract}
The themes that involve economic and social development have received great attention in the present time. Throughout history, different approaches, concepts and measures have been adopted to analyze the development of nations. Among the broader views on the subject is the approach of Amartya Sen, who takes a multidimensional view of the issue, arguing that development encompasses much more than economic growth, including freedoms and the expansion of individual capability. Through the conceptual use proposed by Sen, who considers development as a process of expanding the capability of individuals, this article aims to promote the discussion about the role of the State in the development process.
\end{abstract}

Keywords: State and Development; Capability approach; Amartya Sen 


\section{INTRODUÇÃO}

No decorrer da história da Economia, pode-se encontrar uma diversidade de estudos e conceitos relacionados ao tema desenvolvimento. Dentre tantas abordagens teóricas que utilizam o termo, encontra-se a de Amartya Sen, que considera o desenvolvimento como sendo um processo que possibilita a expansão das capacitações individuais.

Para Sen (1988), definir um conceito para desenvolvimento não é uma tarefa simples. Mas, no decorrer dos anos, as discussões e o conhecimento baseado em trabalhos empíricos realizados possibilitaram esclarecer os problemas subjacentes ao conceito de desenvolvimento. Com a elucidação dos principais problemas inerentes ao conceito de desenvolvimento teve início uma nova visão do assunto, porém sem descartar os conceitos definidos na origem da economia do desenvolvimento.

A teoria tradicional do desenvolvimento, de acordo com Sen (1983), considera como temas principais que influenciam no crescimento econômico: a industrialização, a acumulação de capital, a mobilização de mão de obra, o planejamento e um Estado ativo. Por meio de estudos apresentados por Sen, é possível verificar que esses fatores são importantes para o desenvolvimento econômico, de modo que a teoria tradicional não pode ser invalidada. Porém, Sen acredita que essa teoria apresenta certas limitações, dentre elas o fato de não perceber que o crescimento econômico é apenas um meio para atingir outros objetivos e que a importância está nos benefícios gerados nesse processo de crescimento.

Na percepção de Sen, o desenvolvimento abrange muito mais do que o crescimento econômico e a disponibilidade de bens e serviços em uma economia; o desenvolvimento envolve as liberdades individuais e a possibilidade de escolher individualmente sobre o uso dos bens para a expansão das capacitações individuais. Assim, esse autor adota um conceito multidimensional para o desenvolvimento.

Considerando esta abordagem, o artigo parte do seguinte problema: é possível “desenhar" um papel para o Estado no processo de alcance do desenvolvimento baseado no conceito de Amartya Sen? A hipótese inicialmente levantada, com base em Sen, é de que o Estado, formado por diferentes agentes e instituições, pode atuar no desenvolvimento de tal forma que proporcione a expansão das capacitações dos indivíduos.

O objetivo deste estudo é promover a discussão sobre o papel do Estado no processo de desenvolvimento, sendo este compreendido como um caminho para a expansão das capacitações individuais. O artigo apresenta, além dessa introdução, um breve histórico do 
conceito de desenvolvimento até chegar à abordagem de Sen (seção 1); o enfoque específico da abordagem das capacitações (seção 2), e a relação entre Estado e desenvolvimento (seção 3). Ao final do artigo, são apresentadas algumas considerações sobre o tema em questão.

\section{A TEMÁTICA DO DESENVOLVIMENTO ECONÔMICO}

Todaro e Smith (2009) argumentam que o desenvolvimento econômico é um dos mais novos, emocionantes e difíceis ramos da Economia. Apesar de considerarem que "A Riqueza das Nações" de Adam Smith, publicada em 1776, foi um tratado sobre o desenvolvimento econômico, ao mesmo tempo afirmam que, na África, Ásia e América Latina, o estudo sistemático das questões relacionadas ao assunto só teve início nas últimas cinco décadas, configurando-se como uma área recente de estudo.

Para Kang (2011), a preocupação de muitos economistas com o subdesenvolvimento teve seu auge no período após a Segunda Guerra Mundial, quando surgiu um novo campo de estudos denominado como "Development Economics". Kang (2011) apresenta ainda alguns estudiosos como marcos importantes das modernas teorias de desenvolvimento econômico do pós-guerra, por exemplo, Paul Rosenstein-Rodan, Albert Otto Hirschman, William Arthur Lewis, Ragnar Nurkse e Gunnar Myrdal. Afirma que dentre esses autores é unânime a defesa por políticas de aceleração do crescimento com alguma intervenção governamental.

Inicialmente, a análise de desenvolvimento econômico era baseada na medida de crescimento econômico, ou seja, o aumento de renda per capita era o índice utilizado como medida de avaliação. O crescimento, por sua vez, segundo Campos (2012), até a exposição das ideias de Solow em 1956, era visto como resultado da combinação apenas dos fatores capital e trabalho. A partir de então, nas palavras do autor: "Solow demonstrou que apenas uma pequena parcela poderia ser atribuída a estes fatores, o restante seria atribuído ao resíduo, que seria a medida de nossa ignorância" (CAMPOS, 2012, p. 2). Ou seja, Solow chegou à conclusão de que o crescimento estava relacionado aos fatores capital e trabalho, porém existiriam outros fatores "desconhecidos" que apresentavam importante influência no processo de crescimento. A partir daí, ocorre uma maior abertura na visão dos diversos estudiosos com relação ao crescimento e ao desenvolvimento.

Nos estudos de Lucas (1988) é possível identificar uma distinção entre desenvolvimento e crescimento. Nesta visão, o crescimento está relacionado aos fenômenos 
sobre os quais se têm conhecimento, enquanto que o desenvolvimento refere-se aos fenômenos ainda não compreendidos.

\begin{abstract}
It seems to be universally agreed that the model I have just reviewed is not a theory of economic development. Indeed, I suppose this is why we think of 'growth' and 'development' as distinct fields, with growth theory defined as those aspects of economic growth we have some understanding of, and development defined as those we don't (LUCAS, 1988, p. 13).
\end{abstract}

De acordo com Campos (2012), dá-se então um importante passo nos estudos sobre crescimento, na medida em que a tecnologia e o capital humano passam a ser reconhecidos como determinantes do crescimento econômico.

Assim, é possível identificar que a fonte da teoria do desenvolvimento está nos estudos sobre crescimento econômico, podendo justificar o que Sen (1988) considera como uma estreita ligação entre o desenvolvimento econômico e o crescimento econômico. Essa ligação, apesar de ser uma questão importante, é também geradora de certa confusão, quando o desenvolvimento passa a ser condicionado unicamente ao crescimento econômico. Sen (1988) afirma que realmente existe uma relação entre a expansão das riquezas e as melhorias nas condições de vida das pessoas, porém esse fato isolado não representa totalmente o desenvolvimento.

A abordagem de Sen abrange uma amplitude maior de questões para definir o desenvolvimento. Apesar de não descartar a construção tradicional do conceito, Sen identifica limitações na teoria tradicional. De acordo com Campos (2012), a abordagem de Sen revelase como uma evolução importante no pensamento econômico sobre o desenvolvimento, que passa a receber maior destaque no final do século XX.

Para Evans (2002), Sen conseguiu demonstrar que a renda é uma medida inadequada para avaliar o bem-estar da população, e que o argumento utilitarista reduz a noção de bemestar a um bem-estar homogêneo, na medida em que não considera, por exemplo, as questões valorativas individuais, as quais, para Sen, têm importante influência na definição de bemestar.

Observa-se em Sen (1983) uma importante análise crítica com relação à teoria tradicional do desenvolvimento, na medida em que essa teoria não reconhece que o crescimento econômico é apenas um meio para atingir outros objetivos. 
recognition that economic growth was no more than a means to some other objectives (SEN, 1983, p. 753).

Além disso, o autor afirma que entre as deficiências temáticas da Economia do desenvolvimento tradicional, a principal é o fato de concentrar a atenção no produto interno, na renda agregada e na oferta total de bens particulares, ao invés de considerar os direitos e capacitações das pessoas, ou seja, focar naquilo que as pessoas conseguem ou não fazer. Sen (1983) considera ainda que a análise do crescimento pela renda fornece apenas a informação de que a pessoa tem o meio para adquirir algo, mas não garante que a pessoa poderá exercer todas as suas capacitações e direitos, mesmo que tenha renda disponível para isso. Como pode ser confirmado em Sen (1983, p. 756): "But this rise in income may not be able to deal at all adequately with his entitlement to education or medical treatment (...)”.

De acordo com a abordagem de Sen (1983), alguns pontos específicos limitam a teoria tradicional na análise do desenvolvimento econômico. Um dos limitadores é o fato de a Economia tradicional não tratar o crescimento econômico como apenas um meio das pessoas realizarem suas capacitações, o que, para Sen, é característica essencial do desenvolvimento. Outro fator que cerca a visão econômica tradicional é a análise do desenvolvimento econômico baseada no PIB per capita que poderia ser complementada com a análise dos direitos e capacitações das pessoas. Como uma terceira limitação, Sen (1983) considera que a análise pela produção per capita pode ser falha, na medida em que a distribuição de bens pode ocorrer indevidamente. Utilizando como exemplo a oferta de alimentos, é possível ocorrer que, mesmo com uma oferta suficiente de alimentos, possam existir pessoas passando fome.

Todaro e Smith (2009), ao compararem a Economia do desenvolvimento com a teoria neoclássica tradicional e com a Economia Política, acreditam que a primeira deve preocuparse, em maior grau do que as outras duas, com exigências econômicas, culturais e políticas, de maneira a alcançar com maior eficiência os frutos do progresso econômico para a população, em uma abrangência mais ampla possível.

Para Todaro e Smith (2009), a Economia tradicional assume um caráter materialista e individualista das decisões econômicas, preocupando-se com a alocação eficiente de recursos escassos, com menores custos de produção e a busca por um crescimento ótimo no longo prazo. A Economia Política excede a tradicional, na medida em que abrange o estudo dos processos sociais e institucionais, analisa as relações de poder na economia e o impacto das ações dos grupos, "elites" econômicas e políticas, na alocação de recursos para benefício próprio ou mesmo da população em geral. O desenvolvimento econômico, segundo Todaro e 
Smith, ultrapassa os outros dois conceitos, alcança uma abrangência ainda maior que a Economia Política. Além de o desenvolvimento econômico preocupar-se com a alocação eficiente de recursos, com o crescimento sustentado ao longo do tempo, também analisa questões econômicas, políticas, sociais, institucionais, questões de nível público e privado, voltadas à melhoria dos níveis de vida das diversas populações do mundo.

Thus development economics, to a greater extent than traditional neoclassical economics or even political economy, must be concerned with the economic, cultural, and political requirements for effecting rapid structural and institutional transformations of entire societies in a manner that will most efficiently bring the fruits of economic progress to the broadest segments of their populations (TODARO; SMITH, 2009, p. 8).

Mantendo essa ideia central, Sen $(1983,1988)$ acredita que os estudos relacionados ao desenvolvimento devem ir além de questões puramente econômicas e levar também em consideração os fatores políticos e sociais que afetam as capacitações dos indivíduos.

Segundo Sen (1988), uma expansão de riqueza, identificada pelo crescimento do Produto Interno Bruto (PIB), a princípio poderia representar uma contribuição na melhora das condições de vida das pessoas. Porém, a análise do PIB representa apenas um crescimento econômico, sem abranger as condições de vida da população. Sen (1988) acredita que questões como vestuário, alimentação, habitação, saúde e educação também devem ser consideradas para se avaliar as condições de vida das pessoas. Assim, o autor apresenta cinco fatores principais que diferenciam crescimento e desenvolvimento econômico.

De acordo com Sen (1988), a primeira diferença entre crescimento e desenvolvimento econômico está no fato de que, por meio da análise do PIB, um país pode ser considerado em crescimento econômico, mas a distribuição de renda, de serviços e de produtos pode ser inadequada. Nessa visão, os "benefícios" do crescimento não abrangem e não se distribuem para toda a população de um país. Outra diferença é que o PIB considera apenas os meios de bem-estar que tenham um valor de transação no mercado.

A terceira diferença elencada por Sen (1988) é que o PIB pode representar uma valorização exagerada das commodities, pode distorcer a importância relativa das mercadorias de um país. Em quarto lugar, o autor destaca a questão da temporalidade, alerta para o fato de que o rendimento apreciado por uma pessoa em um determinado período não refletirá as suas condições de vida e bem-estar ao longo da vida.

Por fim, o quinto tópico apresentado por Sen (1988), referente ao crescimento e desenvolvimento, é que o PIB representa apenas uma medida dos meios através dos quais as 
pessoas poderão buscar o bem-estar, porém não denota que as pessoas envolvidas realmente estão conseguindo se utilizar desses meios.

Segundo Todaro e Smith (2009), ainda que existam benefícios "econômicos" relacionados a um determinado padrão de vida que possa ser considerado "bom" aos indivíduos, existirão também custos "não-econômicos" em manter-se nesse padrão. Uma família que vive com um alto padrão de renda, possivelmente sofre pressões competitivas relacionadas ao sucesso financeiro que poderão influenciar negativamente em algumas capacitações relacionadas, por exemplo, ao lazer e ao desfrutar de uma vida saudável. Nesse caso, não ocorrerá plenamente a expansão das capacitações desses indivíduos.

Sen (1988) assume que existem fatores que dificultam a definição de um conceito de desenvolvimento, como o próprio sentido valorativo do termo; considera que o desenvolvimento está diretamente relacionado à noção de valores que possam permiti-lo ocorrer. $\mathrm{O}$ autor enumera dois pontos que evidenciam como o conceito de avaliação de valores se torna uma dificuldade para definir o desenvolvimento. O primeiro é o fato de que os valores são individuais, ou seja, variam de pessoa para pessoa e, em segundo lugar, acrescenta que as mudanças que ocorrem em um processo de desenvolvimento provocam alterações nas avaliações das pessoas envolvidas, ou seja, o próprio processo de desenvolvimento gera mudanças valorativas. Para Sen (1988) "estes dois problemas podem ser denominados, respectivamente, de 'valor de heterogeneidade' e 'valor de endogeneidade"".

Todaro e Smith (2009) também argumentam que o conceito de desenvolvimento contém implícita e explicitamente a influência de valores individuais, inclusive relacionados aos objetivos que um indivíduo deseja alcançar. Por isso, a validade da análise econômica deve ser realizada à luz de valores acordados por uma nação, por meio dos representantes responsáveis pela tomada de decisão no país.

\section{A ABORDAGEM DAS CAPACITAÇÕES DE AMARTYA SEN}

De acordo com Nasar (2012), nas décadas de 70 e 60, Amartya Sen sugeriu uma teoria geral do bem-estar como forma de associar a tradicional preocupação dos economistas em relação ao bem-estar material com a preocupação filosófica sobre direitos individuais e justiça. Para Nasar (2012), Sen apresentou objeções à crença utilitarista de alguns 
economistas, os quais utilizavam o crescimento do PIB per capita como principal medida para avaliar o progresso de um país.

Nasar (2012) afirma que Sen defendeu a liberdade, e não a riqueza, como a verdadeira medida do progresso de uma sociedade. Esta foi considerada um fim primário assim como o principal meio para alcançar o desenvolvimento econômico, uma ideia embasada em uma longa tradição que percorreu de Aristóteles à Friedrich Von Hayek e John Rawls.

Mesmo ciente da dificuldade de definir um conceito para desenvolvimento, Sen (2010) constrói sua abordagem na qual a liberdade é considerada o principal meio e o principal fim para alcançar o desenvolvimento.

\begin{abstract}
A expansão das liberdades é vista, por essa abordagem, como o principal fim e o principal meio do desenvolvimento. O desenvolvimento consiste na eliminação de privações de liberdade que limitam as escolhas e as oportunidades das pessoas de exercer ponderadamente sua condição de agente. A eliminação de privações de liberdades substanciais, argumenta-se aqui, é constitutiva do desenvolvimento (SEN, 2010, p. 9).
\end{abstract}

Sen (2010) relaciona o desenvolvimento à liberdade individual, de modo que essa ligação excede uma relação constitutiva. Ele considera a influência de oportunidades econômicas, das liberdades políticas e dos poderes sociais sobre aquilo que as pessoas conseguem realizar; também abrange as condições habilitadoras, que permitem seus funcionamentos, como boa saúde, educação e incentivos e aperfeiçoamento de iniciativas. Em sua abordagem, defende o papel dos indivíduos como agentes no processo de desenvolvimento, e não como seres passivos apenas beneficiários desse processo.

Sen (1990) afirma que as capacitações de um indivíduo refletem as várias combinações de funcionamentos que ele pode alcançar, o que envolve uma concepção de vida sobre como uma combinação de diversos modos de ser. A capacitação, para Sen (1990), reflete a liberdade individual de escolha por um modo de viver.

De acordo com Sen (1988), a abordagem das capacitações possibilita uma estratégia diferente de valoração voltada para a avaliação da vida, considera-a fundamental ao conceito de desenvolvimento. Essa abordagem acaba por conceber o desenvolvimento de forma multidimensional, considera os diversos ramos da vida que influenciam no individual e na sociedade.

Todaro e Smith (2009) defendem a concepção de desenvolvimento de forma multidimensional, afirmam que se trata de um processo de grandes mudanças nas estruturas sociais, envolve atitudes populares e de instituições nacionais, assim como crescimento 
econômico, redução de desigualdades sociais e erradicação da pobreza. Os autores consideram que o desenvolvimento, essencialmente, deve representar toda a gama de mudanças por meio das quais um todo social afasta-se de uma situação considerada amplamente insatisfatória, para aproximar-se de uma situação na qual as condições de vida tanto no âmbito material quanto no espiritual são consideradas melhores. Todaro e Smith (2009) consideram Amartya Sen como sendo provavelmente o melhor pensador sobre o significado do desenvolvimento, visto da ótica multidimensional.

De acordo com Porsse e Klering:

\begin{abstract}
A Abordagem das Capacitações, desenvolvida por Amartya Sen (1985a, 1985b, 1985c, 1996, 2000, 2001), consiste em um amplo modelo normativo para a análise de arranjos sociais, bem-estar, qualidade de vida, padrões de vida, desigualdade, pobreza, justiça e para o desenho e avaliação de políticas e propostas de mudança social, tendo sido construída a partir de questionamentos da forma de entender, conceber e medir o bem-estar na tradição utilitarista. Dessa forma, a Abordagem das Capacitações busca uma superação do conceito tradicional de bem-estar utilitarista, preocupando-se não com os meios que uma pessoa tem, mas, com os fins que uma pessoa alcança, ou seja, com as realizações dos indivíduos (PORSSE; KLERING, 2008, p. 141).
\end{abstract}

Segundo Sen $(1985,1988)$, a capacitação de um indivíduo pode ser considerada como um conjunto de funcionamentos que ele pode escolher realizar. Comer, falar, pensar, escutar, pode servir como exemplos de funcionamentos. Para Sen (1988), essa liberdade de escolha de opções de funcionamentos pode diferir para cada indivíduo, diferenciando o nível de bemestar que cada um poderá atingir; um pode ter maiores opções de escolhas enquanto o outro pode sofrer alguma privação. Sendo assim, o autor defende que a importância da liberdade na determinação do bem-estar está em vê-lo como uma função não só da realização de um funcionamento efetivo, mas também do conjunto de capacitações a partir do qual o indivíduo escolheu os funcionamentos que desejou realizar. Nessa escolha está refletido o valor dado pelo indivíduo ao conjunto de funcionamentos que ele procurou realizar.

Essa liberdade de escolha de um modo de vida, que reflete os valores do indivíduo e proporciona a expansão das suas capacitações é que, para Sen, pode-se considerar o desenvolvimento. Para ilustrar, Sen (1985) utiliza o exemplo de duas pessoas que passam fome, porém, enquanto uma é privada da alimentação a outra escolhe passar fome por questões religiosas:

To illustrate, consider two persons with identical actual functioning vectors, including--as it happens - both of them starving. Person A is starving because she is very poor and lacks the means to command food. Person B is starving out of choice, 


\begin{abstract}
because of his religious beliefs, which have made him decide to starve and undergo the consequent suffering. In terms of the misery caused by the starvation, we learn that there is no difference between A's experience and B's. Even if it were plausible to say that A and B both have the same level of well-being, in terms of being undernourished, miserable, etc (that complex issue will be examined presently), there would nevertheless remain an important difference between the two cases, viz., B could have in a straightforward sense, chosen an alternative life style which A could not have chosen (SEN, 1985, p. 201).
\end{abstract}

Sen (1988) defende que, partindo da ótica de que o desenvolvimento está relacionado à conquista de uma vida melhor, ao analisá-lo deve-se manter o foco na natureza da vida que as pessoas conseguem viver e nos funcionamentos que conseguem realizar. Sob esta ótica, a avaliação do desenvolvimento incluirá a expectativa de vida das pessoas, as suas liberdades de alimentação, mobilidade, participação social, dentre outros fatores que Sen classifica como "elementos funcionais de uma pessoa".

A abordagem de Sen (1983) defende que a renda e as mercadorias servem como meios para atingir o desenvolvimento, na medida em que podem oportunizar a expansão das capacitações dos indivíduos, o que para Sen é a base do processo de desenvolvimento econômico.

The process of economic development can be seen as a process of expanding the capabilities of people. Given the functional relation between entitlements of persons over goods and their capabilities, a useful - though derivative - characterization of economic development is in terms of expansion of entitlements (SEN, 1983, p. 755).

Por fim, o foco da abordagem de Sen está na análise de o que as pessoas podem ou não realizar, ao que as pessoas conseguem fazer utilizando-se da renda e dos insumos disponíveis em uma economia, e não está ligada unicamente à quantidade dessa renda ou desses insumos. Conforme Sen (1983, p. 755), "ultimately, we are concerned with what people can or cannot do, and this links directly with their 'entitlements' rather than with overall supplies and outputs in the economy”.

Para Sen (2010), as ações dos agentes que compõem o Estado e as instituições sociais são fundamentais para analisar o processo de manutenção e expansão das capacitações dos indivíduos de uma sociedade. Ao considerar os indivíduos como participantes ativos, capazes de interferir no processo de desenvolvimento, Sen (2010) entende que as pessoas não devem exercer apenas um papel passivo (de beneficiários) das ações de desenvolvimento. Assim, o Estado desenvolve um importante papel nesse processo de desenvolvimento identificado por Sen. 


\title{
3 ESTADO E DESENVOLVIMENTO
}

Conforme Chang (1994), o papel do Estado na economia representa uma das questões mais controversas desde a origem da disciplina. De acordo com o autor, existe, entre os estudiosos do tema, uma ideia geral de que o Estado tem um importante papel a desempenhar. Porém não há um consenso com relação ao momento e à maneira como deve ocorrer a participação estatal na economia.

\begin{abstract}
The role of the state in the capitalist economy has been one of the most controversial issues in economics since the birth of the discipline (Deane, 1989). Almost everyone agrees that the state has a role to play, but there is little agreement as to when and how it should act. Perhaps the reason why there is such little agreement is that state intervention is a complex phenomenon involving many contentious issues such as efficiency, morality, power, liberty and legitimacy, to name just a few (CHANG, 1994, p. 7).
\end{abstract}

Pessali e Dalto (2010) confirmam a característica controversa do papel do Estado no desenvolvimento econômico. Os autores apontam a existência de diversos estudos que evidenciam a importância do papel do Estado nas economias desenvolvidas. Ao assumir o papel de agente transformador, o Estado apresentou-se como um determinante às economias em catching-up.

\footnotetext{
Isso se deu principalmente por meio do planejamento, da organização, da coordenação e do financiamento de uma estratégia de mudanças maiores do que as que poderiam ser engendradas por estratégias de firmas individuais ou de mercados (esses, aliás, por definição não podem ser formuladores de estratégia) (PESSALI; DALTO, 2010, p. 30).
}

A ambiguidade da questão do Estado na economia pode ocorrer devido às alterações nos contextos históricos. Em cada período da história é possível perceber diferentes visões com relação à participação do Estado na economia. Segundo Evans (1996), na fase inicial da teoria do desenvolvimento, entre os anos 50 e 60, o Estado era considerado de fundamental importância para o fomento da industrialização. Além disso, representava um papel importante para modernização da agricultura e como suporte para a infraestrutura necessária à urbanização que ocorria no contexto.

Porém, de acordo com o autor, nas décadas posteriores, o Estado perde a imagem de agente transformador e passa a ser visto como um obstáculo ao crescimento. Conforme 
Campos (2012), nas décadas de 1980 e 1990, imperou o ideário liberal, que surge em resposta a um Estado que foi considerado ineficiente. Neste contexto, defende-se certa "limitação" da ação estatal.

O conjunto de medidas adotadas podia variar, mas normalmente passava pela proteção da propriedade privada, implicando na privatização de estatais, estabilidade monetária, cumprimento de contratos, liberalização comercial e financeira, disciplina fiscal, e uma série de medidas para tornar as economias mais expostas aos incentivos de mercado (CAMPOS, 2012, p. 4).

Para Bresser-Pereira (2006, p. 214), a liderança do desenvolvimento econômico é exercida pelo governo, cúpula dirigente do Estado, que atua via acordos com os agentes econômicos no processo de definir políticas econômicas e instituições que gerem investimentos e crescimento da produtividade. Segundo o autor, desde o surgimento do Estado moderno, existe a preocupação do Estado com relação ao desenvolvimento. Afirma ainda, que cabe ao Estado "estabelecer o equilíbrio entre o desenvolvimento e a distribuição, entre lucros e salários e entre os investimentos e as despesas sociais". Esse autor também acredita que a nação orienta as ações dos agentes econômicos por meio da instituição de uma estratégia de desenvolvimento.

\footnotetext{
Uma estratégia nacional de desenvolvimento, porém, é sempre uma ação efetivamente concertada de uma nação; é uma instituição através da qual a nação orienta a ação dos agentes econômicos nacionais e reduz a incerteza; (...) (BRESSER-PEREIRA, 2006, p. 215).
}

Segundo Chang (2004), com relação ao papel do Estado, o processo de desenvolvimento exige a socialização dos riscos dos investimentos inerentes a esse processo, salientando que não necessariamente isso ocorra via políticas diretas, como proteção tarifária ou subsídios, mas também por meio de instituições que socializem esse risco. Porém, deve-se levar em conta o fato de que as instituições podem não ter eficácia ao tratar de problemas relativos a indústrias isoladas e também que constituição de instituições exige tempo, o que pode limitar a reação dos países no tempo adequado.

Para Chang (2004, p. 209), “em muitos casos, uma política mais enfocada e ágil pode ser preferível a soluções institucionais”. O autor apoia a participação do Estado e das instituições no processo de desenvolvimento, desde que ocorram de forma adequada ao contexto do país. Considera que os países atualmente desenvolvidos contaram com o apoio do 
Estado e das devidas instituições em seu processo de desenvolvimento, com políticas condizentes com as necessidades do contexto no qual esse processo ocorreu.

De acordo com Pessali e Dalto (2010), o Estado tem a responsabilidade de estabelecer, manter e fazer cumprir um sistema jurídico que coordene e regule as atividades das demais instituições. É neste grupo seleto de instituições que ocorre a concentração da discussão sobre o desenvolvimento econômico.

Para Sen (2010), apoiado em sua abordagem multidimensional de desenvolvimento, existe a necessidade de buscar um equilíbrio entre Estado, mercado, instituições políticas e sociais, defende o "caminho do meio" entre o livre mercado e a intervenção Estatal.

\footnotetext{
Vale a pena considerar simultaneamente a eficiência por meio da liberdade do mecanismo de mercado, de um lado, e a gravidade dos problemas de desigualdade de liberdade, de outro. É preciso lidar com os problemas de equidade, especialmente ao se tratar de graves privações e pobreza; nesse contexto, a intervenção social, incluindo o custeio governamental pode ter papel importante (SEN, 2010, p. 160).
}

Além disso, segundo Sen (1988), para a realização dos seus funcionamentos, os indivíduos dependem não apenas de "mercadorias" de sua propriedade, mas também da disponibilidade de bens públicos, bens comuns a todos os indivíduos, assim como bens privados disponibilizados pelo Estado, por exemplo, o acesso à saúde e a educação. Nesse caso, o fornecimento destes serviços pelo Estado seria uma forma de melhorar os funcionamentos, podendo oportunizar a expansão de algumas capacitações individuais.

\footnotetext{
A expansão das capacitações tem consequências positivas sobre a produção, que por sua vez, tem potencial para ampliar as capacitações. Se houver um ambiente institucional propício, é possível, portanto, o surgimento de um ciclo virtuoso de desenvolvimento (CAMPOS, 2012, p. 11).
}

De acordo com Evans (2010), em um olhar para o século XXI, fomentar o crescimento econômico está diretamente relacionado a possibilitar a expansão das capacitações humanas, na medida em que o crescimento exige que ocorra uma expansão da utilização do atual acervo de ideias e da geração de novas concepções adequadas ao contexto de cada país.

Sen (2010) não descarta a importância e a eficiência do mecanismo de mercado, porém, acredita que alguns elementos que representam contribuições indispensáveis às capacitações humanas nem sempre podem ser vendidos exclusivamente para um indivíduo, ou seja, existem bens que os indivíduos consomem juntos, como, por exemplo, serviços de saúde, prevenção epidemiológica e ações de preservação ambiental. 
Para Sen (2010), pode existir também a figura de um bem "semipúblico", exemplificando com o caso da educação básica. O autor defende que os benefícios da educação básica geram ganhos não apenas ao indivíduo que a está recebendo. Não apenas a pessoa que recebe a educação se beneficia com isso, pois ocorre um efeito transbordamento provocado pela expansão da educação, ocorrendo mudança social e progresso econômico.

\begin{abstract}
O alcance efetivo desses serviços pode requerer atividades cooperativas e a provisão pelo Estado ou autoridades locais. O Estado tem, com efeito, desempenhado um papel fundamental na expansão da educação básica em todo o mundo. A rápida disseminação da alfabetização na história dos países hoje ricos (no Ocidente, no Japão e no restante da Ásia) baseou-se no baixo custo da educação pública combinada e seus benefícios públicos compartilhados (SEN, 2010, p. 172).
\end{abstract}

Campos (2012) acredita que a educação está entre os bens coletivos dos quais cabe ao Estado garantir, pois considera que o setor privado exige retornos rápidos de seus investimentos. Investimentos em bens coletivos, que oportunizam retornos de longo prazo, devem receber o apoio do Estado. Nesse sentido, o Estado apresenta-se como um importante agente ativo, ao possibilitar a expansão das capacitações individuais que, seguindo a abordagem de Sen, terá como resultado o desenvolvimento.

[...] O mercado falha em prover "capital humano", uma vez que o retorno social é substancialmente maior que o retorno privado (ou seja, uma externalidade positiva, gerando um problema de precificação). Também há a distância temporal entre a educação infantil e o impacto na produção. Além disso, para um investidor privado, o investimento em capital físico apresenta menos incertezas que em capital humano. Portanto, o investimento em educação por parte do Estado apresenta-se como uma necessidade (CAMPOS, 2012, p. 9).

Tal afirmação é confirmada na visão de Sen (2010) sobre os bens públicos em geral, mas não especificamente sobre a educação, a qual para Sen é um bem "semipúblico" na medida em que é direcionada a um indivíduo em específico, apesar de gerar resultados de ampla abrangência social.

A base racional do mercado está voltada para os bens privados (como maçãs e camisas), e não como bens públicos (como o meio livre da malária), sendo possível mostrar que pode haver boas razões para o fornecimento de bens públicos, indo além do que os mercados privados promoveriam (SEN, 2010, p. 171).

De acordo com Evans (1996, p. 2), ao reconhecer o papel central do Estado deve-se vislumbrar que a capacidade de ação do Estado vai além da identificação de políticas; a ação 
estatal também abrange a "institucionalização permanente de um conjunto complexo de mecanismos políticos".

Segundo Campos (2012), se o papel do Estado for considerado como ampliador das capacitações de seus cidadãos, deverá buscar, por exemplo, a redução de taxas de desemprego e a transferência de renda, que podem ser obtidos por meio de ações de política industrial, sob a ótica desenvolvimentista. Além disso, o autor defende que outra justificativa para a atuação do perfil desenvolvimentista do Estado na economia está no fato de que até mesmo a busca por melhores condições de saúde e educação pode não ter os efeitos desejados sobre a ampliação das capacitações dos indivíduos se estes, por exemplo, não possuírem emprego.

\section{CONSIDERAÇÕES FINAIS}

No decorrer da história, o conceito de desenvolvimento foi sendo "humanizado", na medida em que foram introduzidos fatores que ultrapassam a análise baseada unicamente na riqueza. Baseado na abordagem de Amartya Sen, este artigo buscou identificar a possibilidade de "desenhar" um papel para o Estado nesse processo de desenvolvimento.

É possível visualizar o Estado, constituído por diferentes indivíduos e instituições, como um agente possibilitador da expansão das capacitações dos indivíduos. É a concepção de um Estado que adote políticas de desenvolvimento não simplesmente para proteção do mercado interno, mas que oportunize a participação dos indivíduos no processo de desenvolvimento global por meio do acesso aos funcionamentos básicos da vida.

Nessa ótica, desenha-se um Estado formado por instituições democráticas e agentes que atuem na economia não apenas como fomentadores industriais, mas que favoreçam a participação e o crescimento das pessoas como atores sociais, oferecendo condições para que possam exercer as suas liberdades, expandir suas capacitações e participar ativamente do processo de desenvolvimento. Isso seria possível, por exemplo, por meio da disponibilidade de serviços de saúde e educação de qualidade. Tal ação poderia trazer importantes reflexos para o desenvolvimento global, com repercussão inclusive no crescimento econômico, criando o que pode ser chamado de "ciclo virtuoso de desenvolvimento".

A análise do conceito de desenvolvimento abordado por Amartya Sen torna possível iniciar uma importante discussão sobre as diversas possibilidades de ação estatal no processo de desenvolvimento. O Estado pode desempenhar um importante papel nesse processo, 
inclusive por meio da adoção de ações que ultrapassem aquelas convencionadas pelos países atualmente desenvolvidos, e provavelmente os resultados obtidos serão perenes e continuamente crescentes.

\section{REFERÊNCIAS BIBLIOGRÁFICAS}

BRESSER-PEREIRA, L. Estratégia Nacional e Desenvolvimento. Revista de Economia Política, v. 26, n. 2 (102), Abr./Jun., 2006.

CAMPOS, M. M. S. Estado Desenvolvimentista e a ampliação das capacitações. In: XV ENCONTRO DE ECONOMIA DA REGIÃO SUL, ANPEC-SUL. Porto Alegre, 2012.

CHANG, H-J. The Political Economy of Industrial Policy. London: MacMillan Press, 1994.

CHANG, H-J. Chutando a escada. São Paulo: UNESP, 2004.

EVANS, P. El Estado como problema y como solucion. Desarrollo Económico, v. 35, n.140, Enero-Marzo, 1996.

EVANS, P. Collective Capabilities, Culture, and Amartya Sen's Development as Freedom. Studies in Comparative International Development, Summer, v. 32, n. 2, p. 54-60, 2002.

EVANS, P. Construção do estado desenvolvimentista do século XXI: possibilidades e armadilhas. Edigheji, Omano (Ed.), p. 37-58, 2010.

KANG, T. H. Justiça e desenvolvimento no pensamento de Amartya Sen. Revista de Economia Política, v. 31, n. 3 (123), p. 352-369, Julho-Setembro, 2011.

LUCAS, R. E. On the Mechanics of Economic Development. Journal of Monetary Economics, v. 22, n. 1, p. 3-42, 1988.

NASAR, S. A Imaginação econômica: gênios que criaram a economia moderna e mudaram a história. $1^{\text {a }}$. ed. São Paulo: Companhia das Letras, 2012.

PESSALI, H.; DALTO, F. A mesoeconomia do desenvolvimento econômico: o papel das instituições. Nova Economia, v. 29, n. 12, p. 11-37, 2010.

PORSSE, M. C. S.; KLERING, L. R. A abordagem das capacitações: um modelo alternativo para as ações públicas. REDES, Santa Cruz do Sul, v. 13, n. 1, p. 137 - 159, Jan./Abr., 2008.

RANIS, G.; STEWART, F. Economic Growth and human development in Latin American. CEPAL Review, 78, p. 6-23, 2002.

SEN, A. Development: Which Way Now? The Economic Journal, v. 93, n. 372, p. 745-762, 1983. 
Well-Being, Agency and Freedom: The Dewey Lectures 1984. The Journal of Philosophy, v. 82, n. 4, p. 169-221, Apr., 1985.

. The Concept of Development. In: Chenery, H., Srinivasan, T. H. (Eds.). Handbook of Development Economics, Elsevier Science Publishers B. V., cap.1, v. 1, p. 10-26, 1988.

. Development as Capability Expansion. In: Griffin K., Knight, J. (Eds.). Human Development and the International Development Strategy for the 1990s. London: MacMillan, 1990.

. Desenvolvimento como liberdade. São Paulo: Cia das Letras, 2010.

TODARO, M. P.; SMITH, S. C. Economic Development. 10. ed. Boston: Pearson Addison Wesley, 2009. 